\title{
Genetics Analysis of Yield and Quality Characters in Chickpea (Cicer arietinum L.) under Rice Based Cropping System
}

\author{
P.L. Johnson*, R.N. Sharma and H.C. Nanda \\ Department of Genetics and Plant Breeding, Indira Gandhi Krishi Vishwavidyalaya, \\ Raipur-492 012, Chhattisgarh, India \\ *Corresponding author
}

\section{Keywords \\ Combining ability, $\mathrm{L} x \mathrm{~T}$ analysis and chickpea}

Article Info

Accepted: 04 April 2018 Available Online: 10 May 2018

\section{A B S T R A C T}

Analysis of variance for combining ability revealed that the variance due to gca were highly significant for all the characters in all the environments except for days to maturity in $E_{1}$, plant height and primary branches plant ${ }^{-1}$ in $E_{1}, E_{2}$ and $E_{3}$, secondary branches, seed yield and harvest index in $E_{2}$ and $E_{3}$, pods plant ${ }^{-1}$ in $E_{1}$ and $E_{2}$, biological yield plant ${ }^{-1}$ in $E_{2}$ and protein content in $\mathrm{E}_{1}$. Similarly variance due to sca were highly significant for all the characters in all the environments except days to maturity in $\mathrm{E}_{1}$, plat height, primary branches plant ${ }^{-1}$ and secondary branches plant ${ }^{-1}$ in $E_{3}$, seed yield plant ${ }^{-1}$ in $E_{2}$ indicated the importance of both additive and non additive gene effect in their expression. The parent JG 14 and JG 16 appeared to be good general combiners for the trait seed yield plant ${ }^{-1}$ in all the three environments. JG 14 and JG 130 for pods plant ${ }^{-1}$ in $\mathrm{E}_{1}$ and $\mathrm{E}_{2}$, JG 16 and JG 130 in $\mathrm{E}_{3}$. For the trait harvest index, JG 14 and JG 16 in $\mathrm{E}_{1}$ and $\mathrm{E}_{2}$, JG 14 and ICCV 96029 in environments $\mathrm{E}_{3}$. The parents Vaibhav and JG 11 also appeared to be good general combiners for the traits protein content in all three environments. For high seed yield plant $^{-1}$ the cross JG 130 x JG 97, JG 14 x JG 97, JG 315 x JG 97, JG 315 x ICCV 96029 were found as better specific combiners in all the three environments. whereas, Indira Chana-1 x JG 97 in $E_{1}$ and $E_{2}$, JG 11 x JG 97 in $E_{1}$, Vaibhav $x$ ICCV 96029 in $E_{1}$ found as better specific combiners for the characters.

\section{Introduction}

Chickpea [Cicer arietinum (L.) $2 n=2 x=16$ ] belongs to genus Cicer, tribe Cicereae, family Fabaceae, and subfamily Papilionaceae. It is commonly called gram, bengal gram or garbanzo bean, is the most important cool season food grain legume in the world after common bean (Phaseolus vulgaris L.) and pea (Pisum sativum L.). Chickpea is the world's third most important food legume crop grown as rainfed in cool and dry climate in semi-arid regions. During the last few decades, due to increasing demand of the food for world's growing population depend to a large extent on the conservation and use of world's remaining plant genetic resources. Chickpea covers about 11.7 million ha area and 9.3 million tones production in over 45 countries of the world. India is the largest chickpea producer accounting a share of about $67 \%$ in global chickpea production with about 9.93 million ha area, 9.53million tones production and productivity of $960 \mathrm{~kg} / \mathrm{ha}$. Distribution of 
chickpea in six states viz., Madhya Pradesh, Rajasthan, Maharashtra, Uttar Pradesh, Karnataka and Andhra Pradesh together contribute $90.2 \%$ of the production and 90.8 $\%$ of the area of the country. Chhattisgarh covers 0.276 million ha area with production 0.213 million tones and productivity of $771 \mathrm{~kg}$ $\mathrm{ha}^{-1}$. (Project Coordinators Report, 2014-15). A parent which transmits genes for lesser number of days to flowering and maturity to its progeny is regarded as a desirable combiner. Thus parental strains with significant and negative gca effects have been consider as desirable combiners.

\section{Materials and Methods}

The experimental material comprised viz. seven lines viz., Vaibhav, Indira Chana-1, JG 315, JG 11, JG 14, JG 16, JG 130 of agronomic base and three testers JG 97, ICCV 96029 and ICCV 96030 for early maturity were crossed as per Line $\mathrm{x}$ Tester mating design fashion (Kempthorn, 1957) to develop $21 \mathrm{~F}_{1}$ during 2014-15. These $\mathrm{F}_{1}$ along with their parents were evaluated with two replication in one row plot during 2015-16. Under following three rice based cropping systems viz. $\mathrm{E}_{1}$ : Cropping System I: after harvest of early rice variety (Danteshwari) CSI, $\mathrm{E}_{2}$ : Cropping System II: after harvest of medium rice variety (Mahamaya). CS-II, $\mathrm{E}_{3}$ : Cropping System III: after harvest of late rice variety (Dubraj) CS-III. The row to row and plant to plant spacing $30 \times 10 \mathrm{~cm}$ maintained at Research cum Instructional farm, Department of Plant Breeding and Genetics, Indira Gandhi Agricultural University, Raipur The recommended packages of practices were adopted to raise the normal crops. Observations on metric traits where recorded on single plant basis on five randomly selected competitive plant of each genotypes from each replication in each cropping system were as observation on days to $50 \%$ flowering, days to maturity, plant height $(\mathrm{cm})$, number of primary branches plant $^{-1}$, pods plant $^{-1}$, Biological yield plant ${ }^{-1}(\mathrm{~g})$, harvest index $(\%)$, 100 seed weight $(\mathrm{g})$, Seed yield plant $^{-1}(\mathrm{~g})$, seed volume $\left(\mathrm{ml} \mathrm{seed}^{-1}\right)$, hydration capacity $\operatorname{seed}^{-1}(\mathrm{~g})$, hydration index, swelling index and protein content $(\%)$ were recorded on plot basis as per the chickpea descriptor developed by ICRISAT-IBPGR- ICARDA (1993). Sprague and Tatum (1942) were the first to develop the concept of combining ability in terms of genetic variation in Maize. Their results confirmed that the general combing ability (GCA) was primarily due to additive effect of genes and specific combining ability (SCA) was due to combined effects of dominance deviations and epiststic interactions. Rojas and Sprague (1952) concluded that the information on the SCA would often be lower in predictive value than the information on the gca. Griffing (1956) applied the concept of GCA and SCA in relation to diallel crossing system while Kempthorne (1957) proposed this concept in line $\times$ tester analysis.

\section{Results and Discussion}

\section{Analysis of variance}

The analysis of variance for line $\mathrm{x}$ tester analysis for the traits under study has been presented in Table 1). The mean sums of square due the genotypes significant of the mean square for parents vs. hybrid are taken to indicate presence of heterosis. Although this method is statistically valid, conclusion regarding heterosis may not be reliable and useful. It is a comparison of mean of the parents as a group with the mean of crosses as group. Significant could result even when mean of crosses as a group is smaller than mean of parental line as a group.

Moreover, when there is significance, it is quite likely that many crosses do not show heterosis but significant is caused by only a 
few highly heterotic crosses. The mean squares due to genotypes were highly significant for all the traits in $E_{1}, E_{2}$ and $\mathrm{E}_{3}$. The mean squares due to parents were highly significant for all the characters in all the environments except plant height and primary branches plant ${ }^{-1}$ in $\mathrm{E}_{1}$ and $\mathrm{E}_{3}$, pods plant ${ }^{-1}$ in $E_{1}$ and $E_{2}$, harvest index and seed yield plant ${ }^{-1}$ in $E_{2}$ and $E_{3}$, protein content in $\mathrm{E}_{1}$ and swelling index in $\mathrm{E}_{2}$.

The highly significant variance due to parent $v s$ crosses recorded for all the characters in all three environments except days to $50 \%$ flowering in $\mathrm{E}_{1}$, days to $50 \%$ flowering, harvest index and seed yield plant ${ }^{-1}$ in $E_{2}$, days to $50 \%$ flowering, plant height and secondary branches plant ${ }^{-1}$ in $\mathrm{E}_{3}$.

Mean square due to line and tester were found to be significant for all the traits in all the environments except days to maturity in all the environments. Primary branches plant ${ }^{-1}$ in $E_{1}$ and $E_{3}$, secondary branches plant ${ }^{-1}$ and yield plant $^{-1}$ in $\mathrm{E}_{2}$ and protein content in $\mathrm{E}_{3}$. Hence, its indicated that line does not appear to behave consistently over different tester in the respective environments.

\section{Analysis of variance for combining ability}

Analysis of variance for combining ability (Table 2) revealed that the variance due to gca were highly significant for all the characters in all the environment except for days to maturity in $\mathrm{E}_{1}$, plant height and primary branches plant ${ }^{-1}$ in $\mathrm{E}_{1}, \mathrm{E}_{2}$ and $\mathrm{E}_{3}$, secondary branches, seed yield and harvest index in $\mathrm{E}_{2}$ and $E_{3}$, pods plant ${ }^{-1}$ in $E_{1}$ and $E_{2}$, biological yield plant ${ }^{-1}$ in $E_{2}$ and protein content in $E_{1}$. Similarly variance due to sca were highly significant for all the characters in all the environments except days to maturity in $\mathrm{E}_{1}$, plant height, primary branches plant $^{-1}$ and secondary branches plant ${ }^{-1}$ in $E_{3}$, seed yield plant $^{-1}$ in $E_{2}$ indicating importance of both additive and non-additive gene effect in their expression.

Result of the analysis revealed that the variance due to gca was lower than sca variance for all most all the characters in all the environments except protein content in $\mathrm{E}_{2}$, primary branches plant $^{-1}$ and secondary branches plant $^{-1}$ in $E_{3}$, suggesting the predominance of non-additive gene action controlling these characters. Dominance genetic variance was larger than additive genetic variance for the characters, these result are supported by ratio of variance to general to specific combining ability $\left(\sigma^{2}\right.$ gca $/ \sigma^{2}$ sca $)$ which was smaller than unity and by the degree of dominance $\left(\sigma^{2} \mathrm{D} / \sigma^{2} \mathrm{~A}\right)$ which take values greater than unity. Therefore, it applied that the inheritance of these traits was controlling by a preponderance of nonadditive gene effect. Such types of gene action clearly indicated that selection of superior plant for these characters should be postponed to latter generation where these characters can be improved by making selection among the recombinants which the segregating population. As the ratio of gca: sca variance more than unit recorded for protein content in $\mathrm{E}_{2}$, primary branches plant $^{-1}$ and secondary branches plant ${ }^{-1}$ in $E_{3}$ further supported by finding degree of dominance and heritability indicated the role of additive genetic variance in their expression. Hence, direct selection for the characters would be advantageous in isolating chickpea genotypes of more protein content and more primary branches and secondary branches. These finding are in general agreement with the finding of Verma and Waldiya (2010), Monpara and Dhamelia (2013). High estimate of heritability in narrow sense were observed for secondary branches plant $^{-1}$ in $E_{1}, E_{2}$ and $E_{3}$, days to $50 \%$ flowering in $\mathrm{E}_{1}$, primary branches plant ${ }^{-1}$ in $\mathrm{E}_{2}$ and $\mathrm{E}_{3}$, harvest index in $E_{2}$ and $E_{3}$, swelling index in $E_{1} E_{2}$ and $E_{3}$ and protein content in $E_{2}$ (Table $3-5)$. 
Table.1 Analysis of variance for $\mathrm{L} x \mathrm{~T}$ for yield, its attributes and quality characters under $\mathrm{E}_{1}, \mathrm{E}_{2}$ and $\mathrm{E}_{3}$ in chickpea

\begin{tabular}{|c|c|c|c|c|c|c|c|c|c|c|c|c|c|c|c|c|}
\hline \multirow{2}{*}{$\begin{array}{l}\text { Source of } \\
\text { variation }\end{array}$} & \multirow[t]{2}{*}{ D.F. } & \multicolumn{15}{|c|}{ Mean sum of squares } \\
\hline & & $\begin{array}{c}\text { Days to } \\
50 \% \\
\text { flowering }\end{array}$ & $\begin{array}{l}\text { Days to } \\
\text { maturity }\end{array}$ & $\begin{array}{l}\text { Plant } \\
\text { height } \\
(\mathrm{cm})\end{array}$ & $\begin{array}{c}\text { Primary } \\
\text { branches } \\
\text { plant }^{-1}\end{array}$ & $\begin{array}{c}\text { Secondary } \\
\text { branches } \\
\text { plant }^{-1}\end{array}$ & $\begin{array}{l}\text { Pods } \\
\text { plant }^{-1}\end{array}$ & $\begin{array}{l}\text { Biological } \\
\text { yield } \\
\text { plant }^{-1}(\mathrm{~g})\end{array}$ & $\begin{array}{l}\text { Harvest } \\
\text { index }(\%)\end{array}$ & $\begin{array}{c}100 \text {-seed } \\
\text { weight }(\mathrm{g})\end{array}$ & $\begin{array}{l}\text { Seed yield } \\
\text { plant }^{-1}(\mathrm{~g})\end{array}$ & $\begin{array}{c}\text { Seed volume } \\
\left(\mathrm{ml} \mathrm{seed}^{-1}\right)\end{array}$ & $\begin{array}{l}\text { Hydration } \\
\text { capacity } \\
\text { seed }^{-1}(\mathrm{~g})\end{array}$ & $\begin{array}{l}\text { Hydration } \\
\text { index }\end{array}$ & $\begin{array}{l}\text { Swelling } \\
\text { index }\end{array}$ & $\begin{array}{l}\text { Protein } \\
\text { content } \\
(\%)\end{array}$ \\
\hline \multicolumn{17}{|c|}{$E_{1}$ : After harvest of early rice variety of $90-110$ days } \\
\hline Genotypes & 30 & $26.55^{* *}$ & $12.190 * *$ & $40.370 * *$ & $6.140^{* *}$ & $376.02 * *$ & $9197.4 * *$ & $881.57 * *$ & $341.26 * *$ & $20.60 * *$ & $301.12 * *$ & $0.02 * *$ & $0.0213 * *$ & $0.0032 * *$ & $3.920 * *$ & $2.160 * *$ \\
\hline Parents & 9 & $58.56^{* *}$ & $29.50 * *$ & 24.97 & 0.254 & $5.17 * *$ & 162.47 & $151.15^{* *}$ & $342.80 * *$ & $13.445^{* * *}$ & $26.87 *$ & $0.0018^{* *}$ & $0.022342 * *$ & $0.000198^{* *}$ & $1.6848 * *$ & 1.09499 \\
\hline hybrids & 20 & $13.48 * *$ & 2.25 & $46.68 * *$ & $2.371 * *$ & $172.56 * *$ & $3560.1 * *$ & $177.62 * *$ & $231.13 * *$ & $13.07 * *$ & $269.77 * *$ & $0.0019 * *$ & $0.02035^{* *}$ & $0.000391 * *$ & $4.45436 * *$ & $2.1739 * *$ \\
\hline $\begin{array}{l}\text { Par Vs } \\
\text { Hyb }\end{array}$ & 1 & 0.035 & $59.32 * *$ & $52.81 * *$ & $134.59 * *$ & $7783.4^{* *}$ & $20325.8 * *$ & $21530.8 * *$ & $2530.08 * *$ & $235.67 * *$ & $3398.34 * *$ & $0.0091 * *$ & $0.03172 * *$ & $0.000009^{* *}$ & $13.5610^{* *}$ & $11.6757 * *$ \\
\hline Line & 6 & $30.43^{*}$ & $5.300 * *$ & 46.31 & 1.431 & $342.98 * *$ & $3832.58 * *$ & $150.4 *$ & $367.58 * *$ & $23.59 * *$ & $355.410 * *$ & $0.0032 * *$ & $0.03488 * *$ & $0.00065^{* *}$ & $8.1970 * *$ & $4.2395^{*}$ \\
\hline Tester & 2 & 4.22 & 0.863 & 33.34 & 2.301 & $205.75^{* *}$ & $4563.94 * *$ & 127.22 & 93.15 & $2.984 * *$ & $86.820 * *$ & $0.0036^{* *}$ & $0.000343^{*}$ & $0.000026^{* *}$ & 2.3092 & 0.2188 \\
\hline $\begin{array}{l}\text { Line } x \\
\text { Tester }\end{array}$ & 12 & $6.57 * *$ & 0.960 & $49.10^{*}$ & 2.853 & $81.82 * *$ & $3256.67 * *$ & $199.63 * *$ & $185.89 * *$ & $9.5 * *$ & $257.440 * *$ & $0.0011^{* *}$ & $0.0160 * *$ & $0.000317 * *$ & $2.9404 * *$ & $1.467 *$ \\
\hline Error & 30 & 1.00 & 1.35 & 16.33 & 0.510 & 0.1448 & 366.95 & 35.064 & 51.041 & 0.3535 & 9.55 & 0.00008 & 0.00006 & 0.000001 & 0.08136 & 0.77683 \\
\hline \multicolumn{17}{|c|}{$E_{2}$ : After harvest of medium rice variety of $111-135$ days } \\
\hline Genotypes & 30 & $32.79 * *$ & $27.66^{* *}$ & $102.14 * *$ & $4.037^{* *}$ & $310.01 * *$ & $6475.9 * *$ & $731.99 * *$ & $239.4^{* *}$ & $22.18 * *$ & $317.15^{* *}$ & $0.023 * *$ & $0.209^{* *}$ & $0.0032 * *$ & $3.45^{* *}$ & $1.898 * *$ \\
\hline Parents & 9 & $78.2721 * *$ & $73.755 * *$ & 20.9032 & 0.11912 & 0.9205 & 65.1731 & 16.2659 & 54.7656 & $134.488 * *$ & 4.076253 & $0.00186^{* *}$ & $0.022556^{* * *}$ & $0.000198 * *$ & 2.123 & $1.386^{*}$ \\
\hline hybrids & 20 & $13.795^{* *}$ & $2.2312 *$ & $51.1851^{* *}$ & $2.83924^{* *}$ & $176.833^{* *}$ & $3915.12 * *$ & $193.9875^{* * *}$ & $333.513 * *$ & $13.0775^{* *}$ & 254.3312 & $0.00168 * *$ & $0.019547 * *$ & $0.00039 * *$ & $3.65876^{* *}$ & $1.692 * *$ \\
\hline $\begin{array}{l}\text { Par Vs } \\
\text { Hyb }\end{array}$ & 1 & 3.4257 & $121.51 * *$ & $1852.51 * *$ & $63.26703 * *$ & $5755.24 * *$ & $115389.4^{* * *}$ & $17933.6 * *$ & 21.6914 & $283.045^{* *}$ & 4391.305 & $0.0174 * *$ & $0.033053^{* *}$ & $0.000003^{*}$ & $11.2353 * *$ & $10.679 * *$ \\
\hline Line & 6 & $30.484 * *$ & $4.493 *$ & 49.540 & $5.271 *$ & $495.255^{* *}$ & 5097.094 & 223.300 & 367.1 & $23.595^{*}$ & 341.309 & 0.0024 & 0.03327 & 0.000659 & $7.3596^{* * *}$ & $1.386^{*}$ \\
\hline Tester & 2 & 5.453 & 0.170 & 33.810 & 2.366 & 68.437 & 2439.246 & 46.908 & 45.441 & 2.983 & 56.443 & 0.0031 & 0.00054 & 0.000027 & 3.0566 & $1.691 * *$ \\
\hline $\begin{array}{l}\text { Line } x \\
\text { Tester }\end{array}$ & 12 & $6.8411 * *$ & 1.443 & $54.900 * *$ & $1.702 *$ & 35.688 & $3570.089 * *$ & $203.84 * *$ & $364.728 * *$ & $9.500 * *$ & $243.823 * *$ & 0.00103 & $0.01558 * *$ & $0.000317 * *$ & $1.9086^{* *}$ & $10.679 * *$ \\
\hline Error & 30 & 0.88064 & 0.96505 & 14.5232 & 0.43312 & 17.9776 & 526.3379 & 57.3844 & 56.8828 & 0.36509 & 12.31574 & 0.000078 & 0.000094 & 0.000001 & 0.16528 & 0.549 \\
\hline \multicolumn{17}{|c|}{$E_{3:}$ After harvest of late rice variety of above $\mathbf{1 3 5}$ days } \\
\hline Genotypes & 30 & $32.09 * *$ & $26.587 * *$ & $60.40^{* *}$ & $1.993^{* *}$ & $262.75^{* *}$ & $684.75^{* *}$ & $462.16^{* *}$ & $255.53^{*}$ & $23.07 * *$ & $163.47 * *$ & $0.019 * *$ & $0.206^{* * *}$ & $0.0031 * *$ & $3.124 * *$ & $2.385^{* * *}$ \\
\hline Parents & 9 & $74.7999 * *$ & $70.1163 * *$ & 10.6922 & 0.254 & 2.8315432 & $310.1801 * *$ & $26.998 * *$ & 88.2691 & $13.45041^{* *}$ & 5.358101 & $0.001545^{* *}$ & $0.02236^{* *}$ & $0.000199 * *$ & $1.819^{* *}$ & $1.365^{*}$ \\
\hline hybrids & 20 & $15.0953 * *$ & $2.49531 * *$ & 64.3968 & 2.371 & 170.12143 & $479.5344^{* * *}$ & $198.412 * *$ & $326.8023 * *$ & $13.07754 * *$ & $103.991 * *$ & 0.0018431 ** & $0.0193^{* *}$ & $0.000387^{* *}$ & $3.294 * *$ & $2.175^{* * *}$ \\
\hline $\begin{array}{l}\text { Par Vs } \\
\text { Hyb }\end{array}$ & 1 & 0.44140 & $91.7343^{* *}$ & 428.097 & $134.59 * *$ & 4454.602 & $8160.025 * *$ & 9653.697** & $335.781 * *$ & $309.6514 * *$ & 2776.236 ** & $0.00773 * *$ & $0.0333 * *$ & $0.000012^{* *}$ & $11.492 * *$ & $15.754 * *$ \\
\hline Line & 6 & $31.151^{*}$ & $5.429 * *$ & $154.37 * *$ & $2.831 * *$ & $475.26 * *$ & 391.651 & $387.821 * *$ & 335.262 & $23.595^{*}$ & 164.049 & $0.00291 *$ & 0.0331 & 0.000634 & $5.978^{*}$ & $4.241^{*}$ \\
\hline Tester & 2 & 1.2388 & 0.667 & 24.300 & 1.340 & 8.588 & 47.012 & 170.346 & 647.832 & 2.984 & 14.553 & $0.00363 * *$ & 0.00026 & 0.000027 & 2.435 & 0.222 \\
\hline $\begin{array}{l}\text { Line } x \\
\text { Tester }\end{array}$ & 12 & $9.3768 * *$ & 1.333 & $26.092 *$ & 0.651 & $44.469 *$ & $595.562 * *$ & $108.385 * *$ & $269.067 * *$ & $9.500 * *$ & 88.860 ** & $0.0010 * *$ & $0.0154 * *$ & $0.000325 * *$ & $2.0942 * *$ & 1.466 \\
\hline Error & 30 & 0.73334 & 0.72311 & 9.77124 & 0.458789 & 11.83804 & 83.76560 & 21.69353 & 120.4268 & 0.364980 & 9.886743 & 0.000074 & 0.000134 & 0.000001 & 0.367 & 0.588 \\
\hline
\end{tabular}


Table.2 Combining ability and genetic components for seed yield, its attributes and quality characters under $E_{1}, E_{2}$ and $E_{3}$ in chickpea

\begin{tabular}{|c|c|c|c|c|c|c|c|c|c|c|c|c|c|c|c|c|}
\hline $\begin{array}{l}\text { Source } \\
\text { of } \\
\text { variation }\end{array}$ & D.F. & $\begin{array}{c}\text { Days to } \\
\mathbf{5 0 \%} \\
\text { flowering }\end{array}$ & $\begin{array}{l}\text { Days to } \\
\text { maturity }\end{array}$ & $\begin{array}{l}\text { Plant } \\
\text { height } \\
(\mathrm{cm})\end{array}$ & $\begin{array}{c}\text { Primary } \\
\text { branches }^{-1} \\
\text { plant }^{-1}\end{array}$ & $\begin{array}{c}\text { Secondary } \\
\text { branches } \\
\text { plant }^{-1}\end{array}$ & $\begin{array}{l}\text { Pods } \\
\text { plant }^{-1}\end{array}$ & $\begin{array}{l}\text { Biological } \\
\text { yield } \\
\text { plant }^{-1}(\mathrm{~g})\end{array}$ & $\begin{array}{c}\text { Harvest } \\
\text { index }(\%)\end{array}$ & $\begin{array}{c}100 \text {-seed } \\
\text { weight }(\mathrm{g})\end{array}$ & $\begin{array}{c}\text { Seed } \\
\text { yield } \\
\text { plant }^{-1}(\mathrm{~g})\end{array}$ & $\begin{array}{c}\text { Seed } \\
\text { volume } \\
\left(\mathrm{ml} \mathrm{seed}^{-1}\right)\end{array}$ & $\begin{array}{l}\text { Hydration } \\
\text { capacity } \text { seed }^{-1}(\mathrm{~g})\end{array}$ & $\begin{array}{l}\text { Hydration } \\
\text { index }\end{array}$ & $\begin{array}{l}\text { Swelling } \\
\text { index }\end{array}$ & $\begin{array}{l}\text { Protein } \\
\text { content } \\
(\%)\end{array}$ \\
\hline \multicolumn{17}{|l|}{$\mathrm{E}_{1}:$ CSI } \\
\hline GCA & 9 & $58.56^{* * *}$ & $29.50 * *$ & 24.97 & 0.254 & $5.17 * *$ & 162.47 & $151.15^{* *}$ & $342.80 * *$ & $13.445^{* *}$ & $26.87^{*}$ & $0.0018^{* *}$ & $0.022342 * *$ & $0.000198^{* *}$ & $1.6848^{* *}$ & 1.09499 \\
\hline SCA & 20 & $13.48^{* *}$ & 2.25 & $46.68 * *$ & $2.371 * *$ & $172.56^{* *}$ & $3560.1 * *$ & $177.62 * *$ & $231.13^{* *}$ & $13.07^{* *}$ & $269.77 * *$ & $0.0019 * *$ & $0.02035^{* *}$ & $0.000391 * *$ & $4.45436^{* *}$ & $2.1739 * *$ \\
\hline Error & 30 & 1.00 & 1.35 & 16.33 & 0.510 & 0.1448 & 366.95 & 35.064 & 51.041 & 0.3535 & 9.55 & 0.00008 & 0.00006 & 0.000001 & 0.08136 & 0.77683 \\
\hline \multicolumn{17}{|c|}{ Genetic components } \\
\hline$\sigma^{2}$ gca & & 1.065 & 0.211 & -0.927 & -0.099 & 19.254 & 94.158 & -6.082 & 4.447 & 0.378 & -3.632 & 0.0002 & 0.0001 & 0.0000025 & 0.2312 & 0.0762 \\
\hline$\sigma^{2}$ sca & & 2.777 & -0.256 & 15.274 & 1.0774 & 30.703 & 1395.5 & 75.11 & 77.453 & 4.554 & 124 & 0.0005 & 0.0082 & 0.000158 & 10874 & 0.3786 \\
\hline $\begin{array}{l}\sigma^{2} \text { gca/ } \\
\sigma^{2} \text { sca }\end{array}$ & & 0.384 & -0.824 & -0.061 & -0.092 & 0.627 & 0.067 & -0.081 & 0.057 & 0.083 & -0.029 & 0.400 & 0.012 & 0.016 & 0.000 & 0.201 \\
\hline$\sigma 2 \mathrm{~A}$ & & 4.260 & 0.844 & -3.708 & -0.396 & 77.016 & 376.632 & -24.328 & 17.788 & 1.512 & -14.528 & 0.001 & 0.000 & 0.000 & 0.925 & 0.305 \\
\hline$\sigma 2 \mathrm{D}$ & & 11.108 & -1.024 & 61.096 & 4.310 & 122.812 & 5582.000 & 300.440 & 309.812 & 18.216 & 496.000 & 0.002 & 0.033 & 0.001 & 43496.000 & 1.514 \\
\hline h2(ns) & & 30.926 & 12.467 & -13.079 & -11.910 & 40.948 & 7.876 & -5.308 & 9.069 & 14.432 & -9.353 & 7.968 & 3.745 & 0.625 & 46.224 & 20.757 \\
\hline \multicolumn{17}{|l|}{$\mathrm{E}_{2}:$ CS-II } \\
\hline GCA & 9 & $78.2721 * *$ & $73.755^{* * *}$ & 20.9032 & 0.11912 & 0.9205 & 65.1731 & 16.2659 & 54.7656 & $134.488^{* *}$ & 4.076253 & $0.00186^{* *}$ & $0.022556^{* *}$ & $0.000198^{* *}$ & 2.123 & $1.386^{*}$ \\
\hline SCA & 20 & $13.795^{* *}$ & $2.2312^{*}$ & $51.1851 * *$ & $2.83924 * *$ & $176.833^{* *}$ & $3915.12 * *$ & $193.9875^{* *}$ & $333.513^{* *}$ & $13.0775^{* *}$ & 254.3312 & $0.00168^{* *}$ & $0.019547 * *$ & $0.00039 * *$ & $3.65876^{* *}$ & $1.692^{* *}$ \\
\hline Error & 30 & 0.88064 & 0.96505 & 14.5232 & 0.43312 & 17.9776 & 526.3379 & 57.3844 & 56.8828 & 0.36509 & 12.31574 & 0.000078 & 0.000094 & 0.000001 & 0.16528 & 0.549 \\
\hline \multicolumn{17}{|c|}{ Genetic components } \\
\hline$\sigma^{2}$ gca & & 1.112 & 0.0887 & -1.322 & 0.211 & 24.615 & 19.808 & -6.874 & 15.845 & 0.3788 & 4.494 & 0.0002 & 0.0001 & 0.0000025 & 0.3299 & 0.1341 \\
\hline$\sigma^{2}$ sca & & 2.888 & 0.2476 & 20.422 & 0.564 & 5.446 & 1457.8 & 75.26 & 95.77 & 4.5525 & 96.691 & 0.0005 & 0.0079 & 0.0001583 & 0.8313 & 0.125 \\
\hline $\begin{array}{l}\sigma^{2} \text { gca/ } \\
\sigma^{2} \text { sca }\end{array}$ & & 0.385 & 0.358 & -0.065 & 0.374 & 4.520 & 0.014 & -0.091 & 0.165 & 0.083 & 0.046 & 0.400 & 0.013 & 0.016 & 0.397 & 1.073 \\
\hline$\sigma 2 \mathrm{~A}$ & & 4.448 & 0.355 & -5.288 & 0.844 & 98.460 & 79.232 & -27.496 & 63.380 & 1.515 & 17.976 & 0.001 & 0.000 & 0.000 & 1.320 & 0.536 \\
\hline ब2D & & 11.552 & 0.990 & 81.688 & 2.256 & 21.784 & 5831.20 & 301.04 & 383.080 & 18.210 & 386.764 & 0.002 & 0.032 & 0.001 & 3.325 & 0.500 \\
\hline h2(ns) & & 26.421 & 2.479 & -9.065 & 37.762 & 60.039 & 2.263 & -6.967 & 42.783 & 13.442 & 10.912 & 6.933 & 0.383 & 0.625 & 73.001 & 43.841 \\
\hline \multicolumn{17}{|l|}{$\mathrm{E}_{3:}$ CS-III } \\
\hline GCA & 9 & $74.7999 * *$ & $70.1163 * *$ & 10.6922 & 0.254 & 2.8315432 & $310.1801 * *$ & $26.998 * *$ & 88.2691 & $13.45041^{* * *}$ & 5.358101 & $0.001545 * *$ & $0.02236^{* *}$ & $0.000199^{* *}$ & $1.819^{* *}$ & $1.365^{*}$ \\
\hline SCA & 20 & $15.0953 * *$ & $2.49531 * *$ & 64.3968 & 2.371 & 170.12143 & $479.5344 * *$ & $198.412 * *$ & $326.8023 * *$ & $13.07754 * *$ & $103.991 * *$ & $0.0018431 * *$ & $0.0193 * *$ & $0.000387 * *$ & $3.294 * *$ & $2.175^{* *}$ \\
\hline Error & 30 & 0.73334 & 0.72311 & 9.77124 & 0.458789 & 11.83804 & 83.76560 & 21.69353 & 120.4268 & 0.364980 & 9.886743 & 0.000074 & 0.000134 & 0.000001 & 0.367 & 0.588 \\
\hline \multicolumn{17}{|c|}{ Genetic components } \\
\hline$\sigma^{2}$ gca & & 0.681 & 0.171 & 6.324 & 0.1434 & 19.74 & -37.62 & 17.069 & 22.248 & 0.3789 & 0.0435 & 0.0002 & 0.0001 & 0.0000005 & 0.2112 & 0.0763 \\
\hline$\sigma^{2} \mathrm{sca}$ & & 4.242 & 0.284 & 7.579 & 0.0422 & 14.91 & 268.65 & 51.521 & 110.23 & 4.5525 & 38.96 & 0.0005 & 0.0077 & 0.000161 & 0.8125 & 0.3792 \\
\hline $\begin{array}{l}\sigma^{2} \text { gca/ } \\
\sigma^{2} \text { sca }\end{array}$ & & 0.161 & 0.602 & 0.834 & 3.398 & 1.324 & -0.140 & 0.331 & 0.202 & 0.083 & 0.001 & 0.400 & 0.013 & 0.003 & 0.260 & 0.201 \\
\hline$\sigma 2 \mathrm{~A}$ & & 2.724 & 0.684 & 25.296 & 0.574 & 78.960 & -150.480 & 68.276 & 88.992 & 1.516 & 0.174 & 0.001 & 0.000 & 0.000 & 0.845 & 0.305 \\
\hline б2D & & 16.968 & 1.136 & 30.316 & 0.169 & 59.640 & 1074.600 & 206.084 & 440.920 & 18.210 & 155.840 & 0.002 & 0.031 & 0.001 & 3.250 & 1.517 \\
\hline h2(ns) & & 16.598 & 5.009 & 72.098 & 46.790 & 57.512 & -39.161 & 28.222 & 47.342 & 12.935 & 0.201 & 8.388 & 0.388 & 0.129 & 48.399 & 20.531 \\
\hline
\end{tabular}


Table.3 Estimation of General Combing Ability (GCA) effect for yield, its attributing and quality characters in chickpea

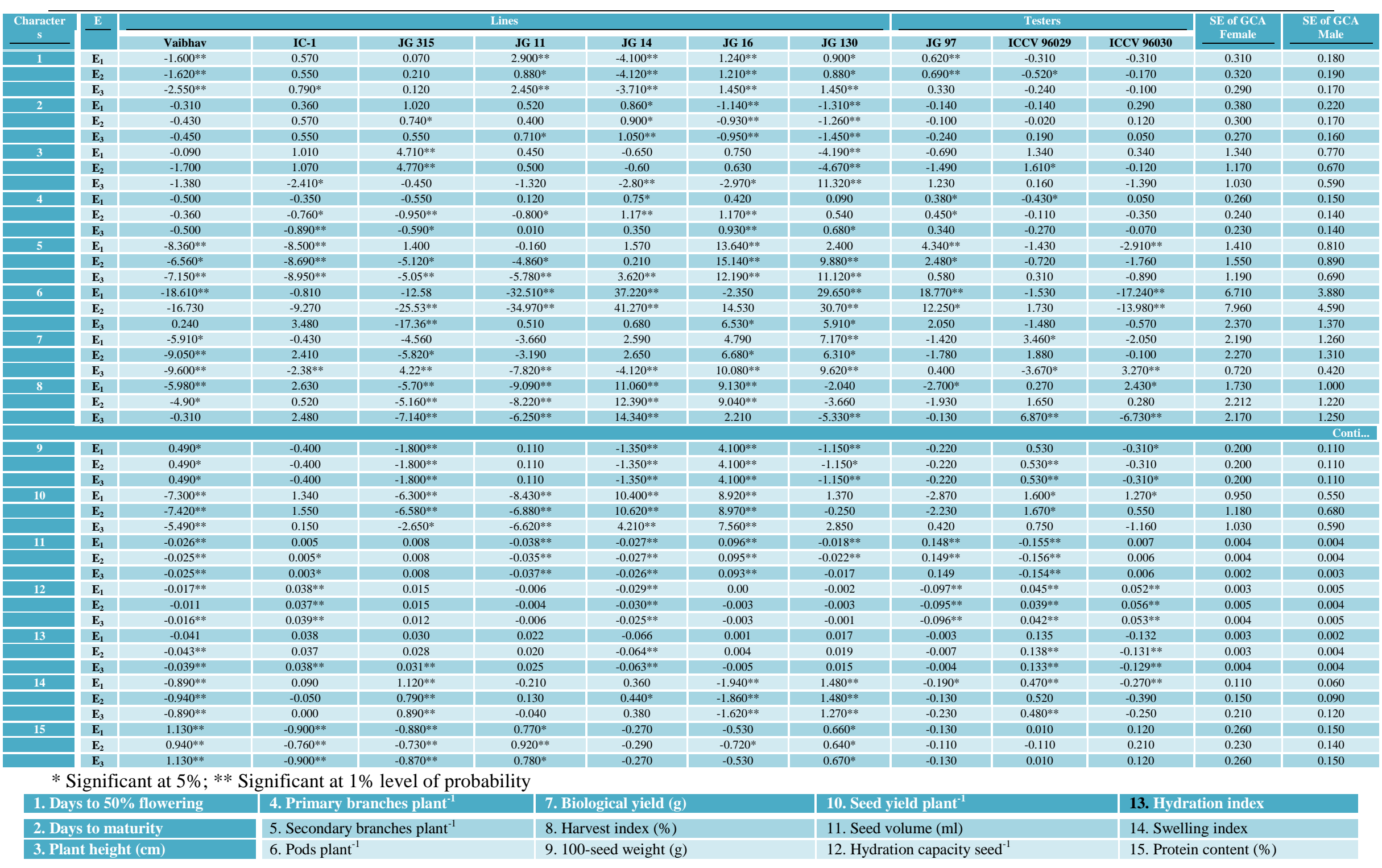


Table.4 Specific Combing ability effects (SCA) of hybrids for seed yield, its attributing and quality characters in chickpea

\begin{tabular}{|c|c|c|c|c|c|c|c|c|c|c|c|}
\hline Hybrids & $\mathbf{E}$ & $\begin{array}{c}\text { Days to } \\
\mathbf{5 0 \%} \\
\text { flowering }\end{array}$ & $\begin{array}{l}\text { Days to } \\
\text { maturity }\end{array}$ & $\begin{array}{c}\text { Plant } \\
\text { height } \\
(\mathrm{cm})\end{array}$ & $\begin{array}{l}\text { Primary } \\
\text { branches } \\
\text { plant }^{-1}\end{array}$ & $\begin{array}{c}\text { Secondary } \\
\text { branches } \\
\text { plant }^{-1}\end{array}$ & $\begin{array}{c}\text { Pods } \\
\text { plant }^{-1}\end{array}$ & $\begin{array}{l}\text { Biological } \\
\text { yield (g) }\end{array}$ & $\begin{array}{c}\text { Harvest } \\
\text { index }(\%)\end{array}$ & $\begin{array}{c}\text { 100-seed } \\
\text { weight } \\
\text { (g) }\end{array}$ & $\begin{array}{l}\text { Seed yield } \\
\text { plant }^{-1}\end{array}$ \\
\hline \multirow[t]{3}{*}{ VAIBHAV x JG 97} & $\mathrm{E}_{1}$ & $-1.120^{*}$ & -0.690 & -1.510 & $0.950 *$ & 1.490 & -1.670 & -1.280 & 4.650 & -0.110 & $2.970^{*}$ \\
\hline & $\mathrm{E}_{2}$ & $-1.020 *$ & $-1.070 *$ & -1.040 & $0.980 * *$ & -0.810 & 6.520 & 0.280 & 2.050 & -0.110 & 1.660 \\
\hline & $\mathrm{E}_{3}$ & $-3.170 * *$ & -0.760 & -0.470 & 0.360 & -1.080 & $8.890 *$ & $-3.830 * *$ & 3.190 & -0.110 & -0.980 \\
\hline \multirow[t]{3}{*}{ VAIBHA V x ICCV 96029} & $\mathrm{E}_{1}$ & -0.290 & 0.140 & -1.910 & 0.550 & -0.380 & $-41.270 * *$ & $-8.220 * *$ & 0.100 & $1.700 * *$ & $-3.560 *$ \\
\hline & $\mathrm{E}_{2}$ & -0.190 & -0.070 & -2.110 & -0.520 & 1.820 & $-46.750 * *$ & -5.780 & -2.490 & $1.700 * *$ & $-4.210^{*}$ \\
\hline & $\mathrm{E}_{3}$ & 0.000 & 0.240 & $-3.560 * *$ & -0.410 & 2.220 & $-8.700^{*}$ & -2.000 & -4.560 & $1.700 * *$ & -3.520 \\
\hline \multirow[t]{3}{*}{ VAIBHAV x ICCV 96030} & $\mathrm{E}_{1}$ & $1.710 * *$ & 0.480 & $-4.210^{*}$ & $-1.450 * *$ & $-8.380 * *$ & $49.700 * *$ & -5.580 & -2.220 & $-2.400 * *$ & $-3.030 *$ \\
\hline & $\mathrm{E}_{2}$ & $1.640 * *$ & -0.240 & $-4.410 *$ & -0.640 & $-4.850 * *$ & 11.720 & -4.950 & -3.800 & $-2.400 * *$ & -3.170 \\
\hline & $\mathrm{E}_{3}$ & $2.170 * *$ & 0.740 & $3.420 * *$ & $-0.860 *$ & -1.280 & $-10.220 * *$ & $4.200 * *$ & -2.460 & $-2.400 * *$ & 2.180 \\
\hline \multirow[t]{3}{*}{ INDIRA CHANA-1xJG-97 } & $\mathrm{E}_{1}$ & -0.120 & -1.020 & -3.750 & -0.510 & $4.490 * *$ & -7.470 & $12.220 * *$ & 0.260 & $2.740 * *$ & $5.200^{*}$ \\
\hline & $\mathrm{E}_{2}$ & -0.020 & $-0.900 *$ & $-3.940 *$ & 0.410 & 0.690 & -12.950 & $16.010 * *$ & 0.680 & $2.740 * *$ & $7.230 *$ \\
\hline & $\mathrm{E}_{3}$ & 0.083 & $-0.930 *$ & 0.590 & 0.590 & $4.250 * *$ & $27.710 * *$ & -1.960 & $6.450 * *$ & $2.740 * *$ & 1.250 \\
\hline \multirow[t]{3}{*}{ INDIRA CH-1x ICCV 96029} & $\mathrm{E}_{1}$ & $-1.620 * *$ & 0.640 & 0.750 & $-1.050^{*}$ & -0.040 & $-50.200 * *$ & $-11.330 * *$ & $-7.180 * *$ & $-2.900 * *$ & $-12.230 * *$ \\
\hline & $\mathrm{E}_{2}$ & $-1.520 * *$ & 0.600 & 0.560 & $-1.450 * *$ & 1.320 & $-44.180 * *$ & $-12.820 * *$ & -3.070 & $-2.900 * *$ & $-10.370 * *$ \\
\hline & $\mathrm{E}_{3}$ & $-1.500 * *$ & -0.260 & $2.870 * *$ & -0.390 & -2.950 & $-28.850 * *$ & $-2.860 *$ & $-12.170 * *$ & $-2.900 * *$ & $-7.180 * *$ \\
\hline \multirow[t]{3}{*}{ INDIRA CH-1x ICCV 96030} & $\mathrm{E}_{1}$ & $-0.950 *$ & 0.640 & $6.750 * *$ & $1.390 * *$ & $-7.310 * *$ & $28.860 * *$ & 4.620 & -1.870 & $1.700 * *$ & 0.950 \\
\hline & $\mathrm{E}_{2}$ & $-1.360 * *$ & $1.430 *$ & $6.720 * *$ & $1.150 * *$ & $-6.310 * *$ & $62.050 * *$ & 5.750 & -4.300 & $1.700 * *$ & 0.480 \\
\hline & $\mathrm{E}_{3}$ & -0.670 & 0.740 & $-4.960 * *$ & 0.280 & -3.210 & $11.500 * *$ & $6.640 * *$ & -3.720 & $1.700 * *$ & 1.170 \\
\hline \multirow[t]{3}{*}{ JG 315 x JG 97} & $\mathrm{E}_{1}$ & $2.380 * *$ & -0.190 & 3.890 & 0.120 & $10.120 * *$ & $22.060 * *$ & $9.580 * *$ & $6.260 * *$ & $-0.750 *$ & $9.700 * *$ \\
\hline & $\mathrm{E}_{2}$ & $2.480 * *$ & 0.260 & $4.220 * *$ & 0.080 & $8.150 * *$ & $23.590 * *$ & 1.510 & $10.920 * *$ & $-0.750 *$ & $8.390 * *$ \\
\hline & $\mathrm{E}_{3}$ & $2.330 * *$ & 0.240 & $2.110 * *$ & 0.430 & 2.050 & -0.430 & -0.200 & $13.280 * *$ & $-0.750 *$ & $7.080 * *$ \\
\hline \multirow[t]{3}{*}{ JG 315 x ICCV 96029} & $\mathrm{E}_{1}$ & $-1.690 * *$ & 0.310 & 0.960 & -0.690 & $-5.740 * *$ & 18.930 & 5.090 & 1.620 & -0.090 & $3.300 * *$ \\
\hline & $\mathrm{E}_{2}$ & $-1.810 * *$ & 0.860 & 0.360 & $-1.060 * *$ & -0.610 & 22.330 & -1.980 & $7.550 *$ & -0.090 & $3.560 * *$ \\
\hline & $\mathrm{E}_{3}$ & -0.600 & 0.810 & -0.700 & -0.230 & 1.690 & $-13.840 * *$ & $3.690 * *$ & $6.700 *$ & -0.090 & $4.490 * *$ \\
\hline \multirow[t]{3}{*}{ JG 315 x ICCV 96030} & $\mathrm{E}_{1}$ & -0.360 & 0.140 & -0.640 & -0.040 & 2.100 & $28.430 * *$ & -2.250 & 3.950 & $-1.600 * *$ & 1.960 \\
\hline & $\mathrm{E}_{2}$ & -0.480 & 0.360 & -0.910 & 0.140 & 0.720 & 25.170 & 0.760 & 1.860 & $-1.600 * *$ & 1.890 \\
\hline & $\mathrm{E}_{3}$ & -0.430 & $-0.190 *$ & $-1.140 * *$ & 0.050 & 1.190 & 2.880 & $-3.130 * *$ & -4.510 & $-1.600 * *$ & $-3.850^{*}$ \\
\hline \multirow[t]{3}{*}{ JG 11 x JG 97} & $\mathrm{E}_{1}$ & -0.360 & -0.020 & $-4.640^{*}$ & 0.360 & 3.600 & -12.800 & $9.240 *$ & 1.380 & -0.200 & $4.800 * *$ \\
\hline & $\mathrm{E}_{2}$ & -0.140 & 0.190 & $-4.910 * *$ & 0.180 & 1.550 & 5.430 & $13.890 * *$ & -5.730 & -0.200 & 2.730 \\
\hline & $\mathrm{E}_{3}$ & -0.260 & -1.190 & $-1.710 * *$ & 0.300 & 1.690 & $15.910 * *$ & $-11.130 * *$ & $15.020 * *$ & -0.200 & 1.650 \\
\hline \multirow[t]{3}{*}{ JG $11 \times$ ICCV 96029} & $\mathrm{E}_{1}$ & 0.310 & 0.480 & $6.020 * *$ & $-1.110 * *$ & -2.240 & -14.170 & $-7.860 * *$ & -0.550 & $-1.060 * *$ & -3.270 \\
\hline & $\mathrm{E}_{2}$ & 0.190 & 0.520 & $5.760 * *$ & -0.420 & -0.110 & -11.430 & $-9.240 * *$ & -1.810 & $-1.060 * *$ & -4.670 \\
\hline & $\mathrm{E}_{3}$ & $-1.100^{*}$ & 0.140 & -1.600 & $-0.800 *$ & -1.980 & $-11.860 * *$ & $6.000 * *$ & $-13.190 * *$ & $-1.060 * *$ & -2.080 \\
\hline
\end{tabular}




\begin{tabular}{|c|c|c|c|c|c|c|c|c|c|c|c|}
\hline \multirow{2}{*}{ Hybrids } & \multirow[b]{2}{*}{$\mathbf{E}$} & \multirow[b]{2}{*}{$\begin{array}{c}\text { Days to } \\
\mathbf{5 0} \% \\
\text { flowering }\end{array}$} & \multirow[b]{2}{*}{$\begin{array}{l}\text { Days to } \\
\text { maturity }\end{array}$} & \multirow[b]{2}{*}{$\begin{array}{c}\text { Plant } \\
\text { height } \\
\text { (cm) }\end{array}$} & \multirow[b]{2}{*}{$\begin{array}{c}\text { Primary } \\
\text { branches }^{-} \\
\text {plant }^{-1}\end{array}$} & \multirow[b]{2}{*}{$\begin{array}{c}\text { Secondary } \\
\text { branches } \\
\text { plant }^{-1}\end{array}$} & \multirow[b]{2}{*}{$\begin{array}{l}\text { Pods } \\
\text { plant }^{-1}\end{array}$} & \multirow[b]{2}{*}{$\begin{array}{l}\text { Biological } \\
\text { yield }(\mathrm{g})\end{array}$} & \multirow[b]{2}{*}{$\begin{array}{l}\text { Harvest } \\
\text { index }(\%)\end{array}$} & \multicolumn{2}{|r|}{ ti...... } \\
\hline & & & & & & & & & & $\begin{array}{c}\text { 100-seed } \\
\text { weight } \\
\text { (g) }\end{array}$ & $\begin{array}{l}\text { Seed yield } \\
\text { plant }^{-1}\end{array}$ \\
\hline \multirow{3}{*}{ JG $11 \times$ ICCV 96030} & $\mathrm{E}_{1}$ & 0.810 & 0.140 & -0.280 & $1.260 * *$ & 0.530 & $-23.100 * *$ & -4.310 & $-13.540 * *$ & $1.050 * *$ & -13.200 \\
\hline & $\mathrm{E}_{2}$ & 0.690 & 0.020 & -0.540 & $1.110^{* *}$ & -1.180 & $-26.870^{* *}$ & -0.780 & $-17.830 * *$ & $1.050^{* *}$ & -13.270 \\
\hline & $\mathrm{E}_{3}$ & $1.070^{*}$ & 0.310 & 0.490 & 0.470 & $7.120^{* * *}$ & 6.480 & $-5.200^{* * *}$ & 2.120 & $1.050 * *$ & -4.010 \\
\hline \multirow{3}{*}{ JG 14 x JG 97} & $\mathrm{E}_{1}$ & 0.480 & -0.360 & -1.280 & -0.710 & $8.760 * *$ & -8.740 & 1.690 & $12.610 * *$ & $1.300 * *$ & $11.280 * *$ \\
\hline & $\mathrm{E}_{2}$ & 0.860 & $-1.140 *$ & -1.380 & $-0.690 *$ & 3.190 & $-25.330 * *$ & -0.510 & $23.400 * *$ & $1.300 * *$ & $12.880 * *$ \\
\hline & $\mathrm{E}_{3}$ & 0.400 & -0.690 & $6.500 * *$ & -0.070 & -2.140 & -2.320 & $3.700 * *$ & $7.240 * *$ & $1.300^{* *}$ & $7.140^{* * *}$ \\
\hline \multirow[t]{3}{*}{ JG 14 x ICCV 96029} & $\mathrm{E}_{1}$ & 0.810 & -0.690 & -0.140 & $0.930^{*}$ & $-7.000 * *$ & 11.460 & -1.600 & -5.470 & 0.600 & $-4.870 * *$ \\
\hline & $\mathrm{E}_{2}$ & 0.690 & -0.810 & 1.620 & $0.740 *$ & -3.550 & 10.700 & 3.860 & $-7.430 * *$ & 0.600 & -3.110 \\
\hline & $\mathrm{E}_{3}$ & $0.900 *$ & 0.810 & $-1.830^{* *}$ & 0.280 & $-7.580^{* *}$ & 2.750 & $6.070^{* *}$ & -13.400 & 0.600 & $-3.350 *$ \\
\hline \multirow{3}{*}{ JG 14 x ICCV 96030} & $\mathrm{E}_{1}$ & $2.810 * *$ & 0.380 & 0.560 & -0.260 & $4.250 * *$ & -17.260 & -3.800 & $-6.280 *$ & 0.200 & $-6.270 * *$ \\
\hline & $\mathrm{E}_{2}$ & $2.830 * *$ & 0.210 & 0.690 & 0.080 & 1.430 & $-28.850 * *$ & 1.700 & $-9.600 * *$ & 0.200 & $-5.220 * *$ \\
\hline & $\mathrm{E}_{3}$ & $3.760 * *$ & -0.050 & $1.170^{* *}$ & -0.130 & -0.610 & 4.850 & 0.140 & $-9.890 * *$ & 0.200 & $-3.500 *$ \\
\hline \multirow[t]{3}{*}{ JG 16 x JG 97} & $\mathrm{E}_{1}$ & 0.640 & -0.290 & 2.560 & -0.510 & -1.720 & 12.840 & $10.460 * *$ & -4.050 & -0.110 & 1.600 \\
\hline & $\mathrm{E}_{2}$ & 0.670 & -0.290 & 3.020 & 0.380 & -2.540 & 21.580 & 5.030 & 0.630 & -0.110 & 2.310 \\
\hline & $\mathrm{E}_{3}$ & 0.430 & -0.050 & $4.700 * *$ & 0.360 & -3.410 & 5.820 & $5.130 * *$ & 9.060 ** & -0.110 & $7.360 * *$ \\
\hline \multirow[t]{3}{*}{ JG 16 x ICCV 96029} & $\mathrm{E}_{1}$ & $-1.360 * *$ & -0.450 & $8.860 * *$ & $1.090 * *$ & 4.780 & $-36.890 * *$ & -3.650 & 0.840 & $2.590 * *$ & -1.770 \\
\hline & $\mathrm{E}_{2}$ & $-1.500 * *$ & 0.050 & $9.320 * *$ & 0.460 & 3.300 & -17.150 & $-8.940 * *$ & $9.530 * *$ & $2.590 * *$ & 0.450 \\
\hline & $\mathrm{E}_{3}$ & $-1.900 * *$ & 0.450 & $-1.710 * *$ & 0.560 & -0.410 & -5.700 & $6.930 * *$ & $-12.560 * *$ & $2.590 * *$ & $-3.840 * *$ \\
\hline \multirow[t]{3}{*}{ JG 16 x ICCV 96030} & $\mathrm{E}_{1}$ & -0.190 & 0.550 & -2.280 & $1.620 * *$ & -2.250 & $21.640 * *$ & -4.350 & 0.300 & $-1.670 * *$ & -1.930 \\
\hline & $\mathrm{E}_{2}$ & -0.170 & 0.380 & -1.810 & 0.010 & -0.570 & $24.380 * *$ & $-6.770 * *$ & 1.140 & $-1.670 * *$ & -2.550 \\
\hline & $\mathrm{E}_{3}$ & 0.260 & 0.790 & $1.010^{*}$ & 0.210 & -2.280 & $-15.860 * *$ & $-4.040 * *$ & $6.740 *$ & $-1.670 * *$ & 0.830 \\
\hline \multirow{3}{*}{ JG 130 x JG 97} & $\mathrm{E}_{1}$ & 0.810 & -0.790 & -0.480 & -0.210 & -0.490 & $73.310 * *$ & $15.650^{* *}$ & $20.720 * *$ & $1.860 * *$ & $25.430 * *$ \\
\hline & $\mathrm{E}_{2}$ & 0.830 & -0.620 & -0.010 & 0.350 & $-0.140 *$ & $71.050 * *$ & $13.600^{* *}$ & $20.900 * *$ & $1.860 * *$ & $23.650 * *$ \\
\hline & $\mathrm{E}_{3}$ & 0.430 & -0.050 & $-3.360 * *$ & -0.080 & $-4.180^{*}$ & $22.370^{* *}$ & $8.060 * *$ & $10.040 * *$ & $1.860 * *$ & $11.200 * *$ \\
\hline \multirow[t]{3}{*}{ JG $130 \times$ ICCV 96029} & $\mathrm{E}_{1}$ & 0.480 & -0.290 & $-5.480 * *$ & -0.680 & -1.450 & $-20.120 * *$ & -6.300 & $-10.740 * *$ & $-3.010 * *$ & $-12.230 * *$ \\
\hline & $\mathrm{E}_{2}$ & 0.500 & -0.290 & -5.350 & -0.450 & 3.130 & -36.720 & 0.760 & $-19.100 * *$ & $-3.010 * *$ & $-13.350 * *$ \\
\hline & $\mathrm{E}_{3}$ & 0.260 & -0.050 & -1.540 & -0.210 & 5.360 & -9.180 & $-10.340 * *$ & -3.520 & $-3.010 * *$ & $-8.300 * *$ \\
\hline \multirow[t]{3}{*}{ JG 130 x ICCV 96030} & $\mathrm{E}_{1}$ & -3.190 & 0.880 & -3.740 & -1.050 & -3.120 & -33.520 & $-7.990 * *$ & -0.790 & 0.140 & $-4.830 * *$ \\
\hline & $\mathrm{E}_{2}$ & -3.170 & 0.550 & -5.850 & -0.820 & -4.600 & -34.290 & -5.370 & -3.490 & 0.140 & $-5.290 * *$ \\
\hline & $\mathrm{E}_{3}$ & -3.240 & -1.050 & -0.280 & -0.710 & 5.520 & -2.310 & $-5.870 * *$ & 0.120 & 0.140 & $-3.740 *$ \\
\hline SE of SCA & $\mathrm{E}_{1}$ & 0.450 & 0.540 & 1.890 & 0.370 & 1.990 & 9.490 & 3.090 & 2.450 & 0.280 & 1.350 \\
\hline SE of SCA & $\mathrm{E}_{2}$ & 0.450 & 0.430 & 1.650 & 0.330 & 2.190 & 11.250 & 3.210 & 3.000 & 0.280 & 1.670 \\
\hline SE of SCA & $\mathrm{E}_{3}$ & 0.420 & 0.390 & 0.330 & 0.330 & 1.680 & 3.360 & 1.020 & 3.070 & 0.280 & 1.460 \\
\hline
\end{tabular}

* Significant at 5\%; ** Significant at $1 \%$ level of probability 
Conti.....

\begin{tabular}{|c|c|c|c|c|c|c|}
\hline Hybrids & $\mathbf{E}$ & Seed volume (ml) & Hydration capacity seed ${ }^{-1}$ & Hydration index & Swelling index & Protein content (\%) \\
\hline \multirow[t]{3}{*}{ VAIBHAV x JG 97} & $\mathbf{E}_{1}$ & $-0.048 * *$ & $0.017 * *$ & $0.074 * *$ & 0.130 & -0.660 \\
\hline & $\mathbf{E}_{2}$ & $-0.046 * *$ & 0.011 & 0.071 & 0.050 & $-0.690 *$ \\
\hline & $\mathbf{E}_{3}$ & $-0.048 * *$ & 0.016 & $0.074 * *$ & 0.040 & -0.660 \\
\hline \multirow[t]{3}{*}{ VAIBHAV x ICCV 96029} & $\mathbf{E}_{1}$ & $0.019 * *$ & -0.010 & -0.100 & 0.220 & -0.010 \\
\hline & $\mathbf{E}_{2}$ & $0.018 * *$ & -0.007 & -0.099 & $-2.280^{*}$ & 0.130 \\
\hline & $\mathbf{E}_{3}$ & $0.018 * *$ & -0.007 & $-0.097 * *$ & 0.200 & -0.020 \\
\hline \multirow[t]{3}{*}{ VAIBHA V x ICCV 96030} & $\mathbf{E}_{1}$ & $0.028 * *$ & -0.007 & $0.026 * *$ & -0.260 & -0.550 \\
\hline & $\mathbf{E}_{2}$ & 0.028 & $-0.004 * *$ & 0.028 & 0.000 & -0.410 \\
\hline & $\mathbf{E}_{3}$ & 0.030 & -0.008 & $0.023 * *$ & 0.040 & -0.550 \\
\hline \multirow[t]{3}{*}{ INDIRA CHANA-1xJG-97 } & $\mathbf{E}_{1}$ & $-0.029 *$ & $-0.038 * *$ & $-0.025 * *$ & $-1.010 * *$ & 0.680 \\
\hline & $\mathbf{E}_{2}$ & $-0.031 * *$ & $-0.037 * *$ & -0.028 & -0.410 & 0.320 \\
\hline & $\mathbf{E}_{3}$ & $-0.027 * *$ & $-0.039 * *$ & $-0.032 * *$ & $-0.910 * *$ & 0.680 \\
\hline \multirow[t]{3}{*}{ INDIRA CHANA-1x ICCV 96029} & $\mathbf{E}_{1}$ & -0.007 & $-0.025 * *$ & $0.066^{* *}$ & $0.900 * *$ & $1.060 * *$ \\
\hline & $\mathbf{E}_{2}$ & -0.007 & $-0.026 * *$ & 0.067 & $0.770 * *$ & $0.870 * *$ \\
\hline & $\mathbf{E}_{3}$ & -0.005 & $-0.023 * *$ & $0.072 * *$ & $0.770 *$ & $1.060 * *$ \\
\hline \multirow[t]{3}{*}{ INDIRA CHANA-1x ICCV 96030} & $\mathbf{E}_{1}$ & $0.037 * *$ & $0.063 * *$ & $-0.041 * *$ & 0.230 & 0.270 \\
\hline & $\mathbf{E}_{2}$ & $0.038 * *$ & $0.062 * *$ & -0.039 & 0.120 & 0.240 \\
\hline & $\mathbf{E}_{3}$ & $0.032 * *$ & $0.061 * *$ & $-0.040 * *$ & -0.040 & 0.270 \\
\hline \multirow[t]{3}{*}{ JG 315 x JG 97} & $\mathbf{E}_{1}$ & $0.028 * *$ & -0.010 & -0.002 & -0.200 & -0.770 \\
\hline & $\mathbf{E}_{2}$ & $0.027 * *$ & $-0.011 * *$ & -0.004 & -0.250 & -0.470 \\
\hline & $\mathbf{E}_{3}$ & $0.024 * *$ & -0.008 & -0.005 & -0.110 & $-0.780 *$ \\
\hline \multirow[t]{3}{*}{ JG 315 x ICCV 96029} & $\mathbf{E}_{1}$ & -0.012 & $0.049 * *$ & $0.035 * *$ & $-0.550 *$ & 0.620 \\
\hline & $\mathbf{E}_{2}$ & -0.012 & 0.051 & 0.036 & -0.440 & $0.740 *$ \\
\hline & $\mathbf{E}_{\mathbf{3}}$ & -0.011 & $0.044 * *$ & $0.039 * *$ & -0.620 & 0.620 \\
\hline \multirow[t]{3}{*}{ JG 315 x ICCV 96030} & $\mathbf{E}_{1}$ & $-0.016^{* *}$ & $-0.038 * *$ & $-0.033 * *$ & $0.430 *$ & -0.120 \\
\hline & $\mathbf{E}_{2}$ & $-0.015 * *$ & $-0.041 * *$ & -0.031 & $0.510^{*}$ & -0.330 \\
\hline & $\mathbf{E}_{3}$ & $-0.013 * *$ & $-0.036 * *$ & $-0.034 * *$ & 0.030 & -0.120 \\
\hline \multirow[t]{3}{*}{ JG 11 x JG 97} & $\mathbf{E}_{1}$ & $-0.033 * *$ & 0.007 & 0.031 & $-1.970 * *$ & 0.100 \\
\hline & $\mathbf{E}_{2}$ & -0.033 & 0.005 & 0.028 & $-1.690 * *$ & -0.110 \\
\hline & $\mathbf{E}_{3}$ & $-0.034 * *$ & $0.006 * *$ & $0.031 * *$ & $-1.620 * *$ & 0.100 \\
\hline \multirow[t]{3}{*}{ JG 11 x ICCV 96029} & $\mathbf{E}_{1}$ & 0.028 & -0.010 & 0.002 & $1.880 * *$ & 0.810 \\
\hline & $\mathbf{E}_{2}$ & 0.025 & -0.014 & 0.003 & $1.490 * *$ & 0.600 \\
\hline & $\mathbf{E}_{3}$ & 0.027 & -0.008 & 0.005 & $1.830 * *$ & 0.810 \\
\hline \multirow[t]{3}{*}{ JG 11 x ICCV 96030} & $\mathbf{E}_{1}$ & 0.005 & 0.003 & -0.033 & 0.170 & $-1.320 * *$ \\
\hline & $\mathbf{E}_{2}$ & 0.008 & 0.009 & -0.032 & 0.050 & $-0.870 * *$ \\
\hline & $\mathbf{E}_{3}$ & 0.007 & 0.001 & $-0.036 * *$ & 0.110 & $-1.320 * *$ \\
\hline
\end{tabular}


Conti.....

\begin{tabular}{|c|c|c|c|c|c|c|}
\hline Hybrids & $\mathbf{E}$ & $\begin{array}{l}\text { Seed volume } \\
(\mathrm{ml})\end{array}$ & $\begin{array}{c}\text { Hydration capacity } \\
\text { seed }^{-1}\end{array}$ & $\begin{array}{l}\text { Hydration } \\
\text { index }\end{array}$ & Swelling index & $\begin{array}{c}\text { Protein content } \\
(\%)\end{array}$ \\
\hline \multirow[t]{3}{*}{ JG 14 x JG 97} & $\mathbf{E}_{1}$ & -0.052 & 0.028 & $0.029 * *$ & -0.240 & -0.210 \\
\hline & $\mathbf{E}_{2}$ & -0.053 & 0.029 & 0.028 & -0.170 & -0.090 \\
\hline & $\mathbf{E}_{3}$ & $-0.053 * *$ & $0.023 * *$ & $0.029 * *$ & -0.090 & -0.210 \\
\hline \multirow[t]{3}{*}{ JG 14 x ICCV 96029} & $\mathbf{E}_{1}$ & 0.033 & 0.037 & -0.085 & 0.280 & 0.130 \\
\hline & $\mathbf{E}_{2}$ & 0.033 & 0.037 & -0.082 & 0.240 & 0.080 \\
\hline & $\mathbf{E}_{3}$ & $0.032 * *$ & $0.036 * *$ & -0.082 & 0.360 & 0.130 \\
\hline \multirow[t]{3}{*}{ JG 14 x ICCV 96030} & $\mathbf{E}_{1}$ & 0.019 & -0.065 & $0.056 * *$ & $0.420 *$ & 0.050 \\
\hline & $\mathbf{E}_{2}$ & 0.020 & -0.066 & 0.054 & 0.390 & -0.050 \\
\hline & $\mathbf{E}_{3}$ & $0.021 * *$ & $-0.060 * *$ & $0.053 * *$ & 0.580 & 0.050 \\
\hline \multirow{3}{*}{ JG 16 x JG 97} & $\mathbf{E}_{1}$ & 0.146 & -0.009 & $-0.048 * *$ & $-0.650 * *$ & 0.140 \\
\hline & $\mathbf{E}_{2}$ & 0.144 & -0.006 & -0.040 & -0.230 & 0.210 \\
\hline & $\mathbf{E}_{3}$ & $0.148 * *$ & -0.004 & $-0.039 * *$ & -0.230 & 0.130 \\
\hline \multirow[t]{3}{*}{ JG 16 x ICCV 96029} & $\mathbf{E}_{1}$ & -0.072 & -0.017 & $0.034 * *$ & $2.230 * *$ & 0.450 \\
\hline & $\mathbf{E}_{2}$ & -0.072 & -0.016 & 0.030 & $1.690 * *$ & 0.520 \\
\hline & $\mathbf{E}_{3}$ & $-0.070 * *$ & $-0.020 * *$ & 0.019 & $1.580 * *$ & 0.450 \\
\hline \multirow[t]{3}{*}{ JG $16 \times$ ICCV 96030} & $\mathbf{E}_{1}$ & -0.073 & 0.026 & 0.014 & $-0.870 * *$ & $-1.490 * *$ \\
\hline & $\mathbf{E}_{2}$ & -0.072 & 0.022 & 0.011 & $-1.080 * *$ & $-0.920 * *$ \\
\hline & $\mathbf{E}_{3}$ & $-0.078 * *$ & $0.024 *$ & 0.020 & $-0.930 * *$ & $-1.490 * *$ \\
\hline \multirow[t]{3}{*}{ JG 130 x JG 97} & $\mathbf{E}_{1}$ & -0.011 & 0.006 & $-0.059 * *$ & $-1.070 * *$ & 0.270 \\
\hline & $\mathbf{E}_{2}$ & -0.009 & 0.008 & -0.055 & $-0.830 * *$ & 0.000 \\
\hline & $\mathbf{E}_{3}$ & $-0.011 *$ & 0.005 & -0.059 & $-0.880 * *$ & 0.270 \\
\hline \multirow[t]{3}{*}{ JG 130 x ICCV 96029} & $\mathbf{E}_{1}$ & 0.010 & -0.025 & $0.047 * *$ & 0.010 & -0.050 \\
\hline & $\mathbf{E}_{2}$ & 0.014 & -0.025 & $0.045^{* *}$ & 0.050 & -0.150 \\
\hline & $\mathbf{E}_{3}$ & 0.009 & $-0.022 * *$ & 0.045 & 0.130 & -0.050 \\
\hline \multirow{3}{*}{ JG 130 x ICCV 96030} & $\mathbf{E}_{1}$ & 0.000 & 0.018 & $0.012 * *$ & -0.080 & 0.650 \\
\hline & $\mathbf{E}_{2}$ & -0.005 & 0.018 & $0.010 * *$ & 0.010 & 0.380 \\
\hline & $\mathbf{E}_{3}$ & 0.002 & $0.017 * *$ & $0.014 * *$ & -0.250 & 0.640 \\
\hline SE of SCA & $\mathbf{E}_{1}$ & 0.004 & 0.004 & 0.004 & 0.150 & 0.370 \\
\hline SE of SCA & $\mathbf{E}_{2}$ & 0.004 & 0.004 & 0.004 & 0.220 & 0.330 \\
\hline SE of SCA & $\mathbf{E}_{3}$ & 0.004 & 0.004 & 0.005 & 0.300 & 0.370 \\
\hline
\end{tabular}

$*$ Significant at 5\%; ** Significant at $1 \%$ level of probability $\mathrm{E}_{1}=$ CS-I; $\mathrm{E}_{2}=\mathrm{CS}-\mathrm{II} ; \mathrm{E}_{3}=\mathrm{CS}-\mathrm{III}$ 
Table.5 Best general and specific combiners for seed yield and its attributing and quality characters in chickpea under different environment

\begin{tabular}{|c|c|c|c|}
\hline Characters & ENV. & Best general combiners & Best specific combiners \\
\hline \multirow[t]{3}{*}{ Days to $50 \%$ flowering } & $\mathrm{E}_{1}$ & JG 14 and Vaibhav & $\begin{array}{l}\text { JG } 315 \text { x ICCV } 96029 \text { and Indira Chana-1 x ICCV } \\
96029\end{array}$ \\
\hline & $\mathrm{E}_{2}$ & JG 14 and Vaibhav & JG 315 x ICCV 96029 and JG 16 x ICCV 96029 \\
\hline & $\mathrm{E}_{3}$ & JG 14 and Vaibhav & Vaibhav x JG 97 and Indira Chana-1 x ICCV 96029 \\
\hline \multirow[t]{3}{*}{ Days to maturity } & $\mathrm{E}_{1}$ & JG 130 and JG 16 & - \\
\hline & $\mathrm{E}_{2}$ & JG 130 and JG 16 & JG 11 x ICCV 96029 and Vaibhav x JG 97 \\
\hline & $\mathrm{E}_{3}$ & JG 130 and JG 16 & Indira Chana-1 x JG 97 and JG 315 x ICCV 96030 \\
\hline \multirow[t]{3}{*}{ Plant height $(\mathrm{cm})$} & $\mathrm{E}_{1}$ & JG 315 & JG 16 x ICCV 96029 and Indira Chana-1 x ICCV 96030 \\
\hline & $\mathrm{E}_{2}$ & JG 315 & JG 16 x ICCV 96029 and Indira Chana-1 x ICCV 96030 \\
\hline & $\mathrm{E}_{3}$ & JG 130 & JG $14 \times$ JG 97 and JG $16 \times$ JG 96 \\
\hline \multirow[t]{3}{*}{ Primary branches plant ${ }^{-1}$} & $\mathrm{E}_{1}$ & JG 14 and JG 97 & JG 16 x ICCV 96030 and Indira Chana-1 x ICCV 96030 \\
\hline & $\mathrm{E}_{2}$ & JG 16 and JG 14 & Indira Chana-1 x ICCV 96030 and JG 11 x ICCV 96030 \\
\hline & $\mathrm{E}_{3}$ & JG 16 and JG 130 & - \\
\hline \multirow[t]{3}{*}{ Secondary branches plant ${ }^{-1}$} & $\mathrm{E}_{1}$ & JG 16 and JG 97 & JG 315 x JG 97 and JG 14 x JG 97 \\
\hline & $\mathrm{E}_{2}$ & JG 16 and JG 130 & JG 315 x JG 97 \\
\hline & $\mathrm{E}_{3}$ & JG 16 and JG 130 & JG 11 x ICCV 96030 and Indira Chana-1 x JG 97 \\
\hline \multirow[t]{3}{*}{ Pods plant $^{-1}$} & $\mathrm{E}_{1}$ & JG 14 and JG 130 & JG 130 x JG 97 and Vaibhav x ICCV 96030 \\
\hline & $\mathrm{E}_{2}$ & JG 14 and JG 130 & JG 130 x JG 97 and Indira Chana-1 x ICCV 96030 \\
\hline & $\mathrm{E}_{3}$ & JG 16 and JG 130 & Indira Chana-1 x JG 97 and JG 130 x JG 97 \\
\hline \multirow[t]{3}{*}{ Biological yield plant ${ }^{-1}(\mathrm{~g})$} & $\mathrm{E}_{1}$ & JG 130 and JG 16 & $\begin{array}{l}\text { Indira Chana-1 x ICCV } 96029 \text { and Vaibhav x ICCV } \\
96029\end{array}$ \\
\hline & $\mathrm{E}_{2}$ & JG 16 and JG 130 & Indira Chana-1 x ICCV 96029and JG 11 x ICCV 96029 \\
\hline & $\mathrm{E}_{3}$ & JG 16 and JG 130 & JG $11 \times$ JG 97 and JG $130 \times$ ICCV 96029 \\
\hline \multirow[t]{3}{*}{ Harvest index (\%) } & $\mathrm{E}_{1}$ & JG 14 and JG 16 & JG 130 x JG 97 and JG 14 x JG 97 \\
\hline & $\mathrm{E}_{2}$ & JG 14 and JG 16 & JG 14 x JG 97 and JG 130 x JG 97 \\
\hline & $\mathrm{E}_{3}$ & JG 14 and ICCV 96029 & JG 11 x JG 97 and JG 315 x JG 97 \\
\hline
\end{tabular}




\begin{tabular}{|c|c|c|c|}
\hline Characters & ENV. & Best general combiners & Best specific combiners \\
\hline \multirow[t]{3}{*}{ 100-seed weight (g) } & $\mathrm{E}_{1}$ & JG 16 and Vaibhav & Indira Chana-1 x JG 97 and JG 16 x ICCV 96029 \\
\hline & $\mathrm{E}_{2}$ & JG 16 and ICCV 96029 & Indira Chana-1 x JG 97 and JG 16 x ICCV 96029 \\
\hline & $\mathrm{E}_{3}$ & JG 16 and ICCV 96029 & Indira Chana-1 x JG 97 and JG 16 x ICCV 96029 \\
\hline \multirow[t]{3}{*}{ Seed yield plant ${ }^{-1}(\mathrm{~g})$} & $\mathrm{E}_{1}$ & JG 14 and JG 16 & JG 315 x JG 97 and Indira Chana-1 x JG 97 \\
\hline & $\mathrm{E}_{2}$ & JG 14 and JG 16 & JG 315 x JG 97 and Indira Chana-1 x JG 97 \\
\hline & $\mathrm{E}_{3}$ & JG 14 and JG 16 & JG 315 x JG 97 and JG 315 x ICCV 96029 \\
\hline \multirow[t]{3}{*}{ Seed volume $\left(\mathrm{ml} \mathrm{seed}^{-1}\right)$} & $\mathrm{E}_{1}$ & JG 97 and JG 16 & Indira Chana-1 x ICCV 96030 and JG 315 x JG 97 \\
\hline & $\mathrm{E}_{2}$ & JG 97 and JG 16 & Indira Chana-1 x ICCV 96030 and JG 315 x JG 97 \\
\hline & $\mathrm{E}_{3}$ & JG 16 and Indira Chana-1 & Indira Chana-1 x ICCV 96030 and JG 315 x JG 97 \\
\hline \multirow[t]{3}{*}{ Hydration capacity seed ${ }^{-1}(g)$} & $\mathrm{E}_{1}$ & ICCV 96030 and ICCV 96029 & $\begin{array}{l}\text { Indira Chana-1 x ICCV } 96030 \text { and JG } 315 \text { x ICCV } \\
96029\end{array}$ \\
\hline & $\mathrm{E}_{2}$ & ICCV 96030 and ICCV 96029 & $\begin{array}{l}\text { Indira Chana-1 x ICCV } 96030 \text { and JG } 315 \text { x ICCV } \\
96029\end{array}$ \\
\hline & $\mathrm{E}_{3}$ & ICCV 96030 and ICCV 96029 & $\begin{array}{l}\text { Indira Chana-1 x ICCV } 96030 \text { and JG } 315 \text { x ICCV } \\
96029\end{array}$ \\
\hline \multirow[t]{3}{*}{ Hydration index } & $\mathrm{E}_{1}$ & - & $\begin{array}{l}\text { Vaibhav x JG } 97 \text { and Indira Chana-1 x ICCV } \\
96029\end{array}$ \\
\hline & $\mathrm{E}_{2}$ & ICCV 96029 & $\begin{array}{l}\text { Vaibhav x JG } 97 \text { and Indira Chana-1 x ICCV } \\
96029\end{array}$ \\
\hline & $\mathrm{E}_{3}$ & ICCV 96029 and Indira Chana-1 & $\begin{array}{l}\text { Vaibhav x JG } 97 \text { and Indira Chana-1 x ICCV } \\
96029\end{array}$ \\
\hline \multirow[t]{3}{*}{ Swelling index } & $\mathrm{E}_{1}$ & JG 130 and JG 315 & JG 16 x ICCV 96029 and JG 11 x ICCV 96029 \\
\hline & $\mathrm{E}_{2}$ & JG 130 and JG 315 & JG 16 x ICCV 96029 and JG 11 x ICCV 96029 \\
\hline & $\mathrm{E}_{3}$ & JG 130 and JG 315 & JG 11 x ICCV 96029 and JG 11 x ICCV 96029 \\
\hline \multirow[t]{3}{*}{ Protein content (\%) } & $\mathrm{E}_{1}$ & Vaibhav and JG 11 & JG 16 x ICCV 96030 and JG 11 x ICCV 96030 \\
\hline & $\mathrm{E}_{2}$ & Vaibhav and JG 11 & JG 16 x ICCV 96030 and JG 11 x ICCV 96030 \\
\hline & $\mathrm{E}_{3}$ & Vaibhav and JG 11 & JG 16 x ICCV 96030 and JG 11 x ICCV 96030 \\
\hline
\end{tabular}

* $\mathrm{E}_{1}$ : CS-I (Environment 1) $\mathrm{E}_{2}$ : CS-II (Environment 2) and $\mathrm{E}_{3}$ : CS-III (Environment 3) 
This supported the involvement of both additive and non-additive gene effects. Rest of the character showed moderates to low narrow sense heritability over the environments. Similar finding were reported by Khan et al., (2006), Gupta et al., (2007), Verma and Waldiya (2010), Bhatt et al., (2013) and Monpara and Gaikwad (2014).

\section{General combining ability}

\section{Yield and contributing characters}

For the Characters primary branches plant ${ }^{-1}$ parent JG 14 and JG 97 in $\mathrm{E}_{1}$ andE $_{2} \mathrm{JG} 16$ in $E_{2}$ and $E_{3} J G 130$ for $E_{3}$ have sown desirable positive gca effects for the traits and among them JG 14 in $E_{1} J G 16$ in $E_{1} E_{2}$, and $E_{3}$ have been considered as the best combiners for the traits. For the traits JG 16 in all three environments, JG 130 in $\mathrm{E}_{2}$ andE $\mathrm{E}_{3}$ and JG 97 in $E_{1}$ and $E_{2}$ have sown desirable positive gca effects for the traits and among them JG 16has been considered as the best general combiners as it shown the maximum gca effects for the traits. For Pods plant ${ }^{-1}$ the parents JG 14 and JG 97 in $\mathrm{E}_{1}$ and $\mathrm{E}_{2}$ and JG 130 in all the environment i.e. $\mathrm{E}_{1}, \mathrm{E}_{2}$ and $\mathrm{E}_{3}$ have exhibited desirable gca and among these JG 14 in $E_{1}$ and $E_{2}$ positive gca effects for the traits JG 130 in $\mathrm{E}_{3}$ have considered as the best general combiners for the traits. For Biological yield plant ${ }^{-1}$ the parents JG 14 and ICCV 96030 in $\mathrm{E}_{3}, J G 16$ in $\mathrm{E}_{2}$ and $\mathrm{E}_{3}$, JG 130 in all the environments and ICCV 96029 in E1 have exhibited the desirable positive gca among them JG 130 in $E_{1}$ and JG 16 in $E_{2}$ and $E_{3}$ have considered as the best general combiners for the traits. For Harvest Index the traits significant positive gca was observed for JG 14 in $E_{1}, E_{2}$ and $E_{3}, J G 16$ in $E_{1}$ and $E_{2}$, ICCV 96029 in $\mathrm{E}_{3}$ and ICCV 96030 in $\mathrm{E}_{1}$, JG 14 has been identified as the best combiner for the traits in all the environments. For 100 seed weight the trait parent Vaibhav, JG 16 and ICCV 96029 have exhibited the desirable positive gca and among them JG 16 has been found as the best general combiner for seed index in all the three environments and Seed yield plant ${ }^{-1}$ the parents JG 14 and JG 16 in all the three environments. ICCV 96029 in $\mathrm{E}_{1}$ and $E_{2}$ and ICCV 96030 in $E_{1}$ exhibited the desirable gca for the traits parents JG 14 in $\mathrm{E}_{1}$ and $E_{2}$ and JG 16 in $E_{3}$ have been considered as the best general combiners.

\section{Quality characters}

The quality characters like Seed volume the trait parents JG 16 in all the three environments, Indira Chana-1 in $\mathrm{E}_{2}$ and $\mathrm{E}_{3}$ and JG 97 in $E_{1}$ and $E_{2}$ exhibited the positive desirable gca and JG 97 in $\mathrm{E}_{1}$ and $\mathrm{E}_{2}$ and JG 16 in $E_{3}$ have identified as the best general combiners for the trait. For Hydration capacity the trait positive desirable gca was observed for Indira Chana-1, ICCV 96029 and ICCV 96030 in all the environments and ICCV 96030 has been identified as the best general combiner for the hydration capacity. For Hydration index the trait parents Indira Chana-1 and JG 315 in $\mathrm{E}_{3}$ and ICCV 96030 in $E_{2}$ and $E_{3}$ have exhibited positive desirable gca effects. While in $E_{1}$ none of the parent has shown significant positive gca. The parent ICCV 96029 has been identified as the best general combiner for hydration index. For Swelling index the trait parents JG 315 and JG 130 in all the three environments have sown positive gca. Similarly, JG 14 in $\mathrm{E}_{2}$ and ICCV 96029 in $E_{1}$ and $E_{3}$ exhibited positive desirable gca for the trait. The parent JG 130 in all the three environments possessed the highest general combining ability effects consider as the best general combiner for the traits in the three environments. For Protein content the positive significant desirable gca has been consider for the parents Vaibhav, JG 11 and JG 16 in all the three environments. Among these parents Vaibhav exhibited the maximum gca and considered as the best general combiner for the trait. Similar results 
were reported by Katiyar et al., (1993), Kamatar et al., (1994), Chaturvedi et al., (1997), Sorde et al., (2000), Jeena and Arora (2001), Bhadouria and Chaturvedi (2003), Kulkarni et al., (2004), Bhatnagar and Singh (2005), Mali et al., (2006), Bhardwaj et al., (2009), Bhardwaj et al., (2010), Bhatt et al., (2013), Mishra et al., (2013), Amdabade et al., (2014) and Monpara and Gaikwad (2014).

\section{Specific combing ability}

Even though SCA effect does not contribute tangibly in the improvement of self-pollinated crops, except in situations where exploration of heterosis is feasible, best crosses are expected to generate transgrasive segregants which could be selected as potent homozygous lines.

Result of sca analysis revealed that some exhibited significant desirable sca estimates in all the environment for one or more characters whereas, for other trait they shared significant SCA in one or two environment. Hence, in order to draw some valid conclusions result of the crosses exhibited significant sca for the traits under all the environments have discussed. The hybrid Vaibhav x JG 97 exhibited desirable negative SCA for days to $50 \%$ flowering in all the three environments. The hybrid Vaibhav x ICCV 96029 exhibited desirable positive SCA in all the environments for 100 seed weight and seed volume. The hybrid Indira chana-1 x ICCV 96029 exhibited desirable negative SCA for days to $50 \%$ flowering and desirable positive SCA for swelling index and protein content in all the environments. The hybrid Indira Chana-1 x ICCV 96030 exhibited desirable positive SCA for pods plant ${ }^{-1}, 100$ seed weight, seed volume and hydration capacity seed $^{-1}$ in all the three environments. The hybrid JG 315 x JG 97 exhibited significant positive SCA for harvest index seed yield plant $^{-1}$ and seed volume in all the three environments. The hybrid JG 315 x ICCV 96029 showed positive desirable SCA for seed yield plant ${ }^{-1}$ in all the three environments i.e., $\mathrm{E}_{1}, \mathrm{E}_{2}$ and $\mathrm{E}_{3}$. The hybrid JG $11 \mathrm{x} \mathrm{ICCV}$ 96029 exhibited desirable positive SCA for swelling index in all the three environments. The hybrid JG 11 x ICCV 96030 exhibited desirable positive SCA for 100 seed weight in all the three environments. The hybrid JG 14 x JG 97 exhibited positive significant desirable SCA for harvest index, 100 seed weight, seed yield plant ${ }^{-1}$ and hydration index in all the three environments. The hybrid JG 16 x ICCV 96029 exhibited desirable negative SCA for days to $50 \%$ flowering whereas, it had desirable positive SCA for plant height, 100 seed weight and swelling index in all the environments. The hybrid JG 130 x JG 97 exhibited desirable positive SCA for pods plant $^{-1}$, biological yield, harvest index, 100 seed weight and seed yield plant ${ }^{1}$ in all the three environments. The hybrid JG $130 \times$ ICCV 96030 exhibited significant positive desirable SCA for hydration index in all the three environments. Similar finding were reported by Khan et al., (2006), Gupta et al., (2007) and Bhatt et al., (2013) for days to maturity. Jayalakshmi et al., (2009) and Naveed et al., (2012) for plant height. Sewak et al., (2012), Gadekar and Dodiya (2013) and Mishra et al., (2013) for primary branches plant $^{-1}$ and Secondary branches plant $^{-1}$. Bhardwaj et al., (2010) for pods plant ${ }^{-1}$, Biological yields, and harvest index, 100 seed weight and seed yield plant ${ }^{-1}$. Naveed et al., (2012)for harvest index, Sidramappa et al., (2008), Malik et al., (2011) and Mishra et al., (2013) for 100 seed weight and seed yield plant $^{-1}$.

\section{Acknowledgement}

The Author are great full to All India Coordinated Research Project on chickpea, Research cum Instructional farm, Department of Genetics and Plant Breeding, Indira Gandhi 
Krishi Vishwavidyalaya, Raipur for providing funding and field for the experiment and also thanks full to Major Advisor Dr. R.N. Sharma, Professor/ Principal Scientist, GPB at this university.

\section{References}

Amadabade, J., Arora, A. and Sahu, H. 2014. Combining ability analysis for yield contributing characters in chickpea (Cicer arietinum L.). Electronic J. Plant Breeding, 5(4): 664-670.

Anonymous, 2014. Project Coordinator's Report, All India Coordinated Research Project on chickpea. Indian Institute Pulses Research, Kanpur, p-29.

Anonymous. 1993. Descriptors for chickpea (Cicer arietinum L.). IBPGR/ ICRISAT/ ICARDA ROME.ICRISAT Patancheru, India, p.1-31.

Bhardwaj, R., Sandhu, J.S. and Singh, I. 2010. Heterosis in relation to combing ability in chickpea (Cicer arietinum L.). Crop Improvement, 37 (2): 126-132.

Bhardwaj, R., Sandhu, J.S., Gupta, S.K. 2009. Gene action and combining ability estimates for yield and other quantitative traits in chickpea. (Cicer arietinum L.). Indian J. Agric. Sci., 79 (11): 897-900.

Chaturvedi, R., I.S. Singh., A.K. Gupta and R. Chaturvedi. 1997. Combining ability analysis in chickpea (Cicer arietinum L.). Agril. Sci. Digest. 17: 1.

Gadekar,

M.S. and Dodiya, N.S. 2013. Heterosis and combining ability analysis for yield and yield contributing traits in chickpea (Cicer arietinum L.). Legume Research, 36 (5): 373-379.

Griffing, B. 1956. Concept of general and specific combining ability in relation to diallel system. Australian. J. Bio. Sci., 9: 463-493.
Gupta, S.K., Sandhu, J.S. and Garg T. 2007. Combining ability in desi chickpea. J. Food Legumes, 20: 22-24.

Gupta, S.K., Sandhu, J.S. and Kumar, A. 2007. Line $\mathrm{x}$ Tester analysis in chickpea (Cicer arietinum L.). Crop Improvement, 34 (2): 170-172.

Jayalakshmi, V., Reddy, C.K.K. and Reddy, M.S. 2009. Heterosis and combining ability in chickpea under moisture stress condition in chickpea (Cicer arietinum L.). J. Food Legumes, 22 (1): 56-58.

Kamatar, M.Y., Biradar, B.D., Hiremath, S.M. 1994. Combining ability studies in chickpea (Cicer arietinum L.). J. Research APAU, 22 (3/4): 97-101

Katiyar R.P., Prasad J. and Katiyar P.K. 1993. Heterosis and combining ability effects in chickpea. Indian J. Pulses Res., 6: 127-131.

Kaur, A., Gupta, S.K. and Singh, K. 2004. Genetic variability in desi chickpea (Cicer arietinum L.) under normal and late sown conditions. J. Res., PAU, 41(4): 425-428.

Kempthorne, O. 1957.An introduction to genetic statistics, New York. John Wiley and Sons, $2^{\text {nd }}$ ed; London: Chapam and Hall, Ltd.

Khan, A.R. 1949. Correlation studies in gram (Cicer arietinum L.). Proc. Agric. for. Sect. $I^{\text {st }}$ Pakistan Sci. Conf. Lahore, pp. 3-4.

Khan, H., Ahmad, F. and Iqbal, N. 2006. Genetic variability and correlations among quantitative traits in gram. Sarhad J. Agric., 22: 55-59.

Kulkarni, S.S., Patil, J.V. and Gawande, V.L. 2004. Heterosis studies in chickpea. (Cicer arietinum L.) J. Maharastra Agricultural University 29 (9): 272-276.

Mali, C.T. Sable, N.B., Wanjari, K.B. and Kalamkar, V. 2006. Combining ability analysis in chickpea (Cicer arietinum L.). J. Phytol. Res., 19 (2): 323-326. 
Monpara, B.A., and Gaikwad, S.R. 2014. Combining high seed number and weight to improve seed yield potential of chickpea. (Cicer arietinum L.). Journal of African Crop Science, 22 (1): 1-7.

Naveed, M.T., Ali, Q., Saeed, U. and Babar, H. 2012. Combing ability analysis for various quantitative traits in chickpea (Cicer arietinum L.). IJBPAS, 1 (4):503-511.
Rojas, B.A. and Sprague, G.F. 1952. A comparison of variance components in corn yield trials: III. General and specific combining ability and their interaction with locations and years. Agronomy Journal, 44: 462-466.

Verma, P. and Waldiya, R.S. 2010. Diallel analysis for nodulation and yield contributing traits in chickpea (Cicer arietinum L.). J. Food Legumes, 23 (2): $117-120$.

\section{How to cite this article:}

Johnson, P.L., R.N. Sharma and Nanda, H.C. 2018. Genetics Analysis of Yield and Quality Characters in Chickpea (Cicer arietinum L.) under Rice Based Cropping System. Int.J.Curr.Microbiol.App.Sci. 7(05): 83-98. doi: https://doi.org/10.20546/ijcmas.2018.705.012 